\title{
Embeddings With Multiple Regularity
}

\author{
Gordana Stojanovic \\ Brown University and Penn State University
}

\begin{abstract}
We introduce $(k, l)$-regular maps, which generalize two previously studied classes of maps: affinely $k$-regular maps and totally skew embeddings. We exhibit some explicit examples and obtain bounds on the least dimension of a Euclidean space into which a manifold can be embedded by a $(k, l)$-regular map. The problem can be regarded as an extension of embedding theory to embeddings with certain nondegeneracy conditions imposed, and is related to approximation theory.
\end{abstract}

\section{Introduction}

Two lines in a Euclidean space are called skew if they are not parallel and do not intersect. A submanifold $M^{n}$ of $\mathbf{R}^{N}$ is said to be totally skew if arbitrary tangent lines to $M$ at any two distinct points are skew. Equivalently, one can define an immersion $f: M^{n} \rightarrow \mathbf{R}^{N}$ to be totally skew if for all $x, y \in M^{n}$ the tangent spaces $d f\left(T_{x} M\right)$ and $d f\left(T_{y} M\right)$ as affine subspaces of $\mathbf{R}^{N}$ have an affine span of maximal possible dimension, that of $2 n+1$.

Totally skew embeddings have been introduced and studied in [10]. Other closely related classes of embeddings into affine and projective spaces defined in terms of mutual positions of tangent spaces at distinct points are skew embeddings and T-embeddings and they have also received a considerable amount of attention, see $[7,8,9,10,16,17,18,19]$.

Another, seemingly less closely related, class of embeddings are so-called $k$-regular maps, and their affine version, introduced by Borsuk in [3]. A continuous map $f: X \rightarrow \mathbf{R}^{N}$ is called $k$-regular (respectively affinely $k-1$ - 
regular) if the images under $f$ of $k$ distinct points are linearly (respectively affinely) independent. ${ }^{1}$ The study of $k$-regular maps was motivated by the theory of Chebyshev approximation. It was conducted by non-algebrotopological methods in [3, 4], while Handel [6, 11, 12, 13, 14, 15] introduced cohomological methods using configuration spaces to obtain various existence and non-existence results. Vassiliev [20] independently studied $k$-regular maps under the name ' $k$-interpolating spaces of functions', using topological methods similar to those of Handel. He was interested in the interpolating properties of a finite dimensional space of continuous functions on a topological space. Namely, he calls a finite dimensional space $L$ of continuous functions on a topological space $M, k$-interpolating if every real-valued function on $M$ can be interpolated at arbitrary $k$ points of $M$ with an appropriate function from $L$. The connection with $k$-regular maps is as follows: the functions $f_{1}, \ldots, f_{N}$ span a $k$-interpolating space of functions if and only if the map $f=\left(f_{1}, \ldots, f_{N}\right)$ is $k$-regular. In other words, $f$ is $k$-regular if and only if we can prescribe values at any distinct $k$ points of $M$ for functions in the span of coordinate functions of $f$.

One of the main questions that arises in the study of all mentioned maps is to find the lowest possible dimension of the target Euclidean space which allows them. For example, for a given manifold $M^{n}$, what is the smallest dimension $N=N\left(M^{n}\right)$ such that $M^{n}$ admits a totally skew embedding in $\mathbf{R}^{N}$ ? As is, this question has been answered for very few manifolds. Results are available only for line, circle and plane: $N\left(\mathbf{R}^{1}\right)=3, N\left(S^{1}\right)=4, N\left(\mathbf{R}^{2}\right)=6$, see [10]. Ghomi and Tabachnikov actually give totally skew embeddings of line, circle and plane in the Euclidean space of minimal possible dimension and these are the only known explicit examples of optimal totally skew embeddings. According to the same authors [10], dimension $n$ submanifolds of $\mathbf{R}^{N}$ are generically totally skew when $N \geq 4 n+1$. This abundance of totally skew embeddings contrasted with the scarcity of available examples points to another object of investigation: finding more of them.

The same question can be asked for $k$-regular maps, and one result, that both Handel [14] and Vassiliev [20] reached, is for instance, that when $k$ is even, $N\left(S^{1}\right)=k+1$, and when $k$ is odd, $N\left(S^{1}\right)=k$. While the result for odd $k$ is almost immediate, to achieve the result for $k$ even, they both used

\footnotetext{
${ }^{1}$ The definitions of both types of $k$-regularity that we adopt here are those of Handel, see for example [15]. The definitions used by other authors are in essence the same, but the role of $k$ differs from one author to another. We adapt all the quoted results to our definition.
} 
nonelementary topological methods, in particular, characteristic classes.

We introduce a class of regular maps, so called $(k, l)$-regular maps, which generalize both totally skew embeddings and affinely $k$-regular maps, and ask the same question of determining minimal dimensional target Euclidean space. This problem can be regarded as an extension of investigations that led to the birth of embedding theory - an extension to the embeddings

with certain prescribed non-degeneracy conditions. The interpretation of $(k, l)$-regular maps in the language of the approximation theory is as follows: it turns out that a smooth map $f=\left(f_{1}, \ldots, f_{N}\right): M^{n} \rightarrow \mathbf{R}^{N}$ on a smooth manifold $M^{n}$ is $(k, l)$-regular if and only if for every function in the span of $1, f_{1}, \ldots, f_{N}$ we can prescribe not only values at any distinct points $x_{1}, \ldots, x_{k}, y_{1}, \ldots, y_{l}$ but directional derivatives as well in any direction at the last $l$ points. Thus, the existence of $(k, l)$-regular maps is equivalent to the possibility of interpolating functions on $M^{n}$ through any $k+l$ points and up to the first order derivatives in arbitrary directions at the last $l$ points. Finally, let us mention that there is an obvious connection with recent work of Arnold and his school, see [1].

In this paper, we generalize existing estimates for totally skew and affinely $k$-regular maps to our class, provide explicit examples in the case of line, circle and plane and determine the minimal target spaces for curves. We only employ non-algebro-topological methods, which leaves plenty of room for further investigations in the topology of these embeddings.

Acknowledgments. I would like to thank my advisor Sergei Tabachnikov for suggesting this problem as well as for his constant support and guidance. I would also like to thank Mohammad Ghomi and Bruce Solomon for interesting discussions and Anatole Katok for continual support during my stay at Penn State University, where this work has been carried out.

\section{Definition of $(k, l)$-regular maps}

We will start with the definition of affine independence. There are many different (albeit equivalent) ways to define affine independence, but we settle with the following one.

Definition 2.1 Affine subspaces $V_{1}, \ldots, V_{k} \subset \mathbf{R}^{N}$ are said to be affinely independent if their affine span has the maximal possible dimension that the 
affine span of affine spaces of respective dimensions can have in any given affine ambient space.

For example, the affine span of two lines may have dimension 1, 2 or 3 depending on their position. The maximal of these is three dimensional, and so any two lines are affinely independent if their affine span is three dimensional. Thus no two lines in $\mathbf{R}^{2}$ are affinely independent.

From now on, when we say that some span is maximal possible we will mean maximal possible regardless of the ambient space. In this terminology, no two lines in $\mathbf{R}^{2}$ will have maximal possible affine span.

One can calculate that if $V_{1}, \ldots, V_{k} \subset \mathbf{R}^{N}$ have dimensions $n_{1}, \ldots, n_{k}$ respectively than they are affinely independent if and only if their affine span has the dimension

$$
\left(n_{1}+1\right)+\ldots+\left(n_{k}+1\right)-1 \text {. }
$$

Definition 2.2 Let $M^{n}$ be an $n$-dimensional manifold. Let $k$ and $l$ be nonnegative integers, not both equal to 0 . We will call a smooth map $f: M^{n} \rightarrow \mathbf{R}^{N}$ $(k, l)$-regular if for every set of distinct points $x_{1}, \ldots, x_{k}, y_{1}, \ldots, y_{l}$ of $M$ and of $l$ tangent lines $L_{i} \subset T_{y_{i}} M, i=1, \ldots, l$ the set of points and lines

$$
f\left(x_{1}\right), \ldots, f\left(x_{k}\right), d f\left(L_{1}\right), \ldots, d f\left(L_{l}\right)
$$

is affinely independent.

Remark 2.1 When $l=0$, the notion of $(k, l)$-regularity coincides with the notion of affine $k-1$-regularity as defined in [15]. On the other hand, a smooth map $f$ is $(0,2)$-regular if and only if it is totally skew. Thus, the notion of $(k, l)$-regularity generalizes both totally skew and (affinely) $k$-regular maps.

We will often say that a manifold is $(k, l)$-regular with the understanding that it is a submanifold of some Euclidean space, and the inclusion map is $(k, l)$-regular.

Definition 2.3 The $(0, l)$-regular maps we will also call l-totally-skew.

Remark 2.2 The following properties are more or less obvious:

- If a map is $(k, l)$-regular then it is also $\left(k^{\prime}, l^{\prime}\right)$-regular, for all $k^{\prime} \leq k$, $l^{\prime} \leq l$. 
- Any $(2,0)$-regular map is one-to-one.

- Any 1-totally-skew map is an immersion.

- Any 2-totally-skew map is nothing else but a totally skew embedding.

- Every restriction of a $(k, l)$-regular map is $(k, l)$-regular.

One may be tempted to adopt a more general definition of regularity: instead of requiring affine independence of a collection of points and lines, one may consider arbitrary dimensional vector subspaces of tangent spaces at certain number of distinct points of $M$ and require the affine independence of their images under $d f$. However, it turns out that this doesn't add any generality to the definition, as stated in the following lemma, which we leave without proof.

Lemma 2.1 Let $M^{n}$ be an $n$-dimensional manifold and fix some positive integers $n_{1}, \ldots, n_{l}$ not greater than $n$. Then, the map $f: M^{n} \rightarrow \mathbf{R}^{N}$ is $(k, l)$ regular if and only if for arbitrary distinct points $x_{1}, \ldots, x_{k}, y_{1}, \ldots, y_{l} \in M$ and arbitrary vector spaces $V_{i} \subset T_{y_{i}} M, \operatorname{dim}\left(V_{i}\right)=n_{i}, i=1, \ldots, l$ the affine spaces

$$
f\left(x_{1}\right), \ldots, f\left(x_{k}\right), d f\left(V_{1}\right), \ldots, d f\left(V_{l}\right)
$$

are affinely independent.

To fix notation we will denote $N_{k, l}=N_{k, l}\left(M^{n}\right)$ to be the least possible integer $N$, such that $M^{n}$ admits a $(k, l)$-regular map into $\mathbf{R}^{N}$.

Remark 2.3 A simple dimension counting argument shows that any $n$ dimensional $(k, l)$-regular manifold requires at least $k+(n+1) l-1$ dimensions of ambient space, immediately yielding that

$$
N_{k, l}\left(M^{n}\right) \geq k+(n+1) l-1 \text {. }
$$

In particular,

$$
N_{k, l}(\mathbf{R}) \geq k+2 l-1 \text {. }
$$

Remark 2.4 Since manifolds are locally Euclidean, it follows that

$$
N_{k, l}\left(M^{n}\right) \geq N_{k, l}\left(\mathbf{R}^{n}\right) .
$$

The following observation will be useful in the proofs. 
Lemma 2.2 An embedding $f: M^{n} \rightarrow \mathbf{R}^{N}$ is not $k, l$-regular if and only if there exists an $(k+(n+1) l-2)$-dimensional affine subspace that touches $M$ at $l$ points and intersects it in additional $k$ points.

Proof. It follows from Lemma 2.1 where $V_{j}$ 's are taken to be full tangent spaces. $\square$.

Henceforth we shall assume that $f$ is always an embedding, simply because in most interesting cases (e.g. whenever $k+l>1),(k, l)$-regular maps actually are embeddings.

\section{Examples and bounds}

The following proposition, together with the last item in Remark 2.2 and the existence of $(k, l)$-regular embeddings of real line (Thm 3.3) implies the existence of $(k, l)$-regular embeddings of any manifold into some Euclidean space, and as a consequence, $N_{k, l}$ is well defined.

Proposition 3.1 Let $M$ and $N$ be $(k, l)$-regular submanifolds in euclidean spaces $U$ and $V$ respectively. Then, the embedding

$f: M \times N \rightarrow(U \otimes V) \oplus(U \otimes \mathbf{R}) \oplus(\mathbf{R} \otimes V), f(x, y)=(x \otimes y, x \otimes 1,1 \otimes y)$

is $(k, l)$-regular.

The next theorem improves on the estimate (1) and provides an upper bound as well. The lower bound is obtained by extending the argument in [4], while the upper bound is obtained similarly as in the case of totally skew embeddings [10].

Theorem 3.1 For any manifold $M^{n}$,

$$
\left[\frac{k}{2}\right] n+\left[\frac{k-1}{2}\right]+(n+1) l \leq N_{k, l}\left(M^{n}\right) \leq(n+1) k+(2 n+1) l-1 .
$$

Moreover, generically any submanifold $M^{n}$ in $R^{(n+1) k+(2 n+1) l-1}$ is $(k, l)$-regular.

Setting $k=0$ and $l=2$ gives us the theorem for totally skew embeddings obtained in [10]. On the other hand, the upper bound for the opposite case, $k=2$ and $l=0$, says simply that $M^{n}$ always embeds in $R^{2 n+1}$, a well known 
fact. The theorem also generalizes the lower bound for affinely $k$-regular maps given in [4].

When the manifold is closed, we have a better (by 1) lower bound in the case of $l$-totally-skew embeddings.

Theorem 3.2 Let $M^{n}$ be a closed manifold. Then,

$$
N_{0, l}\left(M^{n}\right) \geq(n+1) l .
$$

Finally, we give examples of $(k, l)$-regular embeddings of real line, circle and plane. In the case of curves, these embeddings happen to be optimal, deciding $N_{k, l}$ when $n=1$.

Proposition 3.2 1. The map $\gamma: \mathbf{R} \rightarrow \mathbf{R}^{k+2 l-1}$ given by

$$
\gamma(t)=\left(t, t^{2}, \ldots, t^{k+2 l-1}\right)
$$

is $(k, l)$-regular.

2. The map $\gamma: S^{1} \rightarrow \mathbf{R}^{2 k^{\prime}+2 l}$, given by

$$
\gamma(\alpha)=\left(\cos \alpha, \sin \alpha, \cos 2 \alpha, \sin 2 \alpha, \ldots, \cos \left(k^{\prime}+l\right) \alpha, \sin \left(k^{\prime}+l\right) \alpha\right)
$$

is $(k, l)$-regular for $k=2 k^{\prime}+1$ (and hence for $k=2 k^{\prime}$ as well).

3. The map $\gamma: \mathbf{R}^{2} \cong \mathbf{C} \rightarrow \mathbf{C}^{k+2 l-1} \cong \mathbf{R}^{2(k+2 l-1)}$ given by

$$
\gamma(z)=\left(z, z^{2}, \ldots, z^{k+2 l-1}\right)
$$

is $(k, l)$-regular.

Theorem 3.3 One has:

1. $N_{k, l}(\mathbf{R})=k+2 l-1$.

2. $N_{k, l}\left(S^{1}\right)= \begin{cases}k+2 l, & k \text { is even } \\ k+2 l-1, & k \text { is odd. }\end{cases}$ 


\section{Proofs}

Let $i$ be the embedding of $\mathbf{R}^{N}$ into $\mathbf{R}^{N+1}$ as the height 1 hyperplane, that is let

$$
i: \mathbf{R}^{N} \rightarrow \mathbf{R}^{N+1}, i: x \mapsto(x, 1) .
$$

Let $A G_{n}(N)$ denote the (affine Grassmanian) manifold of $n$-dimensional affine subspaces of $\mathbf{R}^{N}$, and $G_{n+1}(N+1)$ be the (Grassmanian) manifold of the $(n+1)$-dimensional subspaces of $\mathbf{R}^{N+1}$. Then, $i$ induces the canonical embedding

$$
A G_{n}(N) \rightarrow G_{n+1}(N+1),
$$

given by assigning to each point $p \in \mathbf{R}^{N}$ the line $\ell(p) \subset \mathbf{R}^{n+1}$ which passes through the origin and $(p, 1)$. We will also call this embedding $i$ without any fear of confusion.

The following fact, which is immediate consequence of the definition, we state as a lemma.

Lemma 4.1 The affine span of the affine subspaces $V_{1}, \ldots, V_{k} \subset \mathbf{R}^{N}$ is maximal possible if and only if the linear span of the corresponding vector subspaces $i\left(V_{1}\right), \ldots, i\left(V_{k}\right) \subset \mathbf{R}^{N+1}$ maximal possible. The dimension of this linear span is one larger than the dimension of the affine span of $V_{1}, \ldots, V_{k}$.

Remark 4.1 We will use notation $\tilde{x}=(x, 1)$ for points $x \in M$, but $\tilde{u}=(u, 0)$ for vectors $u$ tangent to $M$. As a consequence of the previous lemma, it follows that $f: M \rightarrow \mathbf{R}^{N}$ is $(k, l)$-regular if and only if for distinct points $x_{1}, \ldots, x_{k}, y_{1}, \ldots, y_{l} \in M$ and non-zero vectors $u_{j} \in T_{y_{j}} M, j=1, \ldots, l$, the vectors $\tilde{x_{1}}, \ldots, \tilde{x_{k}}, \tilde{y_{1}}, \ldots, \tilde{y}_{l}, \tilde{u_{1}}, \ldots, \tilde{u}_{l} \in \mathbf{R}^{N+1}$ are linearly independent.

Proof of Proposition 3.1. First note that a tangent vector to $f(x, y)$ is of the form $(u \otimes y+x \otimes v, u \otimes 1,1 \otimes v)$ where $u \in T_{x} M$ and $v \in T_{y} N$. Fix arbitrary distinct points $\left(x_{i}, y_{i}\right) \in M \times N, i=1, \ldots, k+l$ and arbitrary non-zero vectors $\left(u_{j}, v_{j}\right) \in T_{\left(x_{j}, y_{j}\right)} M \times N, j=k+1, \ldots, k+l$.

Because of remark 4.1, it suffices to show, after the identification

$$
(U \otimes V) \oplus(U \otimes \mathbf{R}) \oplus(V \otimes \mathbf{R}) \oplus \mathbf{R} \cong(U \oplus \mathbf{R}) \otimes(V \oplus \mathbf{R})
$$

that the vectors in the set

$$
\mathcal{S}=\left\{\tilde{x}_{i} \otimes \tilde{y}_{i} \mid i=1, \ldots, k+l\right\} \cup\left\{\tilde{u}_{j} \otimes \tilde{y}_{j}+\tilde{x}_{j} \otimes \tilde{v}_{j} \mid j=k+1, \ldots, k+l\right\}
$$


are linearly independent.

Since $M$ is $(k, l)$-regular, we know that the vectors in $\mathcal{M}=\left\{\tilde{x}_{i}, \tilde{u}_{j} \mid u_{j} \neq 0\right\}$ are linearly independent and similarly for $\mathcal{N}=\left\{\tilde{y}_{i}, \tilde{v}_{j} \mid v_{j} \neq 0\right\}$. But now we observe that every nontrivial linear combination of vectors in $\mathcal{S}$ would also be a nontrivial linear combination of vectors in

$$
\{e \otimes f \mid e \in \mathcal{M}, f \in \mathcal{N}\},
$$

and that is not possible.

Proof of Theorem 3.1. First we prove the lower bound. We will make use of the following theorem, affine version of which is proved in [4]. For one line proof of the even $k$ case of the theorem stated here, see [6].

Theorem 4.1 (Boltyanski-Ryzhkov-Shashkin) If a $k$-regular map of $\mathbf{R}^{n}$ into $R^{N}$ exists, then $N \geq\left[\frac{k}{2}\right] n+\left[\frac{k+1}{2}\right]$.

Since the inequality holds trivially when $k+l=1$, and $f$ is an embedding when $k+l>1$, we can assume without loss of generality that $M^{n}$ is a $(k, l)$-regular submanifold of $R^{N}$.

Without loss of generality, assume $l>0$; otherwise, there is nothing to prove. Now fix some arbitrary distinct points $y_{1}, \ldots, y_{l} \in M^{n}$, and let $W$ be the affine span of $T_{y_{1}} M, \ldots, T_{y_{l}} M$. The inclusion $M \rightarrow \mathbf{R}^{N}$ induces a map $M^{n}-\left\{y_{1}, \ldots, y_{l}\right\} \rightarrow \mathbf{R}^{N+1} / i(W) \cong \mathbf{R}^{N+1-(n+1) l}$. Since $M^{n}$ is $(k, l)$-regular, the induced map is $k$-regular. Because of Theorem 4.1 we have that

$$
N+1-(n+1) l \geq\left[\frac{k}{2}\right] n+\left[\frac{k+1}{2}\right],
$$

which proves the lower bound.

To prove the upper bound, the strategy is to first embed the manifold $M$ in a large Euclidean space $R^{N}$ as a $(k, l)$-regular submanifold. This can be done, for instance, using Proposition 3.1. Then, one reduces the dimension of the target space by succesive projections to hyperplanes all the while preserving $(k, l)$-regularity.

To do that, we project to any hyperplane, centrally from a point $A$ outside of it. The projected manifold will still be $(k, l)$-regular if we choose $A$ away from $U$, the union of affine spans of all $k+l$-tuples of points of $M$ and $l$ tangent spaces at the last $l$ points. Since $U$ consists of points

$$
\sum_{i=1}^{k} a_{i} x_{i}+\sum_{j=1}^{l} b_{j} y_{j}+\sum_{j=1}^{l} v_{j}
$$


where

$$
a_{i}, b_{j} \in \mathbf{R}, x_{i}, y_{j} \in M^{n}, v_{j} \in T_{y_{j}} M^{n}, \sum_{i=1}^{k} a_{i}+\sum_{j=1}^{l} b_{j}=1
$$

one can calculate using local charts that $\operatorname{dim} U=k(n+1)+l(2 n+1)-1$. Thus, for $N>k(n+1)+l(2 n+1)-1$ one can find $A$ not in $U$ and reduce $N$ by 1 . This proves the upper bound.

To prove that the upper bound is generically true, that is, that every embedding $M^{n} \rightarrow \mathbf{R}^{k(n+1)+l(2 n+1)-1}$ can become $(k, l)$-regular after an arbitrarily small perturbation, one uses Thom's transversality theorem. The proof is exactly the same as in [10], so we omit it.

Proof of Theorem 3.2. Thm 3.2 follows immediately from the next proposition and remark 2.2.

Proposition 4.1 If $M^{n}$ is a closed submanifold of $\mathbf{R}^{(n+1) l-1}$, then there exists a hyperplane tangent to $M$ at $l$ distinct points.

Proof. Without loss of generality, we may assume that $M=M^{n}$ does not belong to an affine hyperplane in $\mathbf{R}^{(n+1) l-1}$; if it does, we are done. Let us denote by $\mathrm{C}(M)$ the convex hull of $M$.

Claim 4.1 There exists a point $x \in \partial \mathrm{C}(M)$ that is not a convex combination of $l-1$ or fewer points of $M$.

Proof. The set of all convex combinations of all $l-1$-tuples of points of $M$

$S=\left\{x=a_{1} y_{1}+\ldots+a_{l-1} y_{l-1} \mid \sum_{i=1}^{l-1} a_{i}=1, a_{i} \geq 0, y_{i} \in M, i=1, \ldots, l-1\right\}$

is a set of dimension $(n+1) l-2-n$, containing $M$. On the other hand, since $M$ does not belong to any hyperplane, neither does $\mathrm{C}(M)$. Therefore, $\mathrm{C}(M)$ is a convex set of dimension $(n+1) l-1$, its boundary $\partial \mathrm{C}(M)$ has dimension $(n+1) l-2$. Thus, there must exist a point $x$ in $\partial \mathrm{C}(M)$, but not in $S$, as claimed.

Consider now the support hyperplane $H$ at $x$. It is a hyperplane through $x$, so that $\mathrm{C}(M)$ is contained in a closed half-space bounded by $H$. Since $M$ is closed, $\mathrm{C}(M)$ is compact, and therefore $x \in \mathrm{C}(M)$. By the theorem of 
Carathéodory, see for example [2], every point in $\mathrm{C}(M)$ is a convex combination of at most $(n+1) l$ points, so

$$
x=a_{1} y_{1}+\ldots+a_{(n+1) l} y_{(n+1) l}, \quad \text { where } \quad \sum_{i=1}^{(n+1) l} a_{i}=1, a_{i} \geq 0
$$

and $y_{i} \in M$ for $i=1, \ldots,(n+1) l$. However, all the points $y_{i}$ with non-zero coefficients $a_{i}$ in the above convex combination must belong to $H$, because otherwise $x$ wouldn't be in $H$. So, it is exactly at those points where $H$, our support hyperplane, touches $M$. And by claim 4.1 there must be at least $l$ of them. Which is exactly what we wanted to prove.

\section{Proof of Proposition 3.2}

\section{The open case.}

We will argue by contradiction. Suppose that the curve

$$
\gamma(t)=\left(t, t^{2}, \ldots, t^{k+2 l-1}\right), t \in \mathbf{R}
$$

is not $(k, l)$-regular. Then there exists a hyperplane $H$,

$$
a_{0}+\sum_{i=1}^{k+2 l-1} a_{i} x_{i}=0
$$

tangent to the curve $\gamma(t)$ at $l$ distinct points and intersecting it in at least $k$ additional points. But this means that the polynomial

$$
f(t)=a_{0}+\sum_{i=1}^{k+2 l-1} a_{i} t^{i}
$$

of degree $k+2 l-1$, has $l$ double and $k$ simple roots. Contradiction.

2. The closed case.

Again, suppose that the curve $\gamma: S^{1} \rightarrow \mathbf{R}^{2 k^{\prime}+2 l}$,

$$
\gamma(\alpha)=\left(\cos \alpha, \sin \alpha, \cos 2 \alpha, \sin 2 \alpha, \ldots, \cos \left(k^{\prime}+l\right) \alpha, \sin \left(k^{\prime}+l\right) \alpha\right)
$$

is not $(k, l)$-regular for $k=2 k^{\prime}+1$. Then, just as in the open case we obtain a function

$$
f(\alpha)=a_{0}+\sum_{i=1}^{k^{\prime}+l} a_{i} \cos i \alpha+\sum_{i=1}^{k^{\prime}+l} b_{i} \sin i \alpha .
$$


having $2 k^{\prime}+2 l+1$ roots on the interval $[0,2 \pi)$ when counted with multiplicites. However, $f(\alpha)$ is a trigonometric polynomial of degree $k^{\prime}+l$, and it is a well known fact (see, for example, [5]) that it can have at most $2\left(k^{\prime}+l\right)$ zeros. Thus, our curve is $(k, l)$-regular, just as we claimed.

3. The plane.

First, we observe that this map is $(k, l)$-regular in the complex sense, that is, that for any given distinct points $z_{1}, \ldots, z_{k}, w_{1}, \ldots w_{l} \in \mathbf{C}$, the points $\gamma\left(z_{1}\right), \ldots, \gamma\left(z_{k}\right), \gamma\left(w_{1}\right), \ldots, \gamma\left(w_{l}\right)$ and complex tangent lines at last $l$ points are affinely independent over $\mathbf{C}$. In other words, there do not exist complex numbers $a_{1}, \ldots, a_{k}, b_{1}, \ldots, b_{l}, \xi_{1}, \ldots, \xi_{l}$, not all equal to zero, so that the following realtions hold:

$$
\sum_{i=1}^{k} a_{i} \gamma\left(z_{i}\right)+\sum_{j=1}^{l} b_{j} \gamma\left(w_{j}\right)+\sum_{j=1}^{l} \xi_{j} \gamma^{\prime}\left(w_{j}\right)=0, \sum_{i=1}^{k} a_{i}+\sum_{j=1}^{l} b_{j}=0
$$

The exact same proof as in the case of real open curves goes through when we replace real coordinates and coefficients with complex ones.

Now, we show $(k, l)$-regularity in the real sense. Suppose therefore, towards a contradiction, that there are distinct points $z_{1}, \ldots, z_{k}, w_{1}, \ldots, w_{l} \in \mathbf{R}^{2}$ and non-zero vectors $\xi_{j} \in T_{w_{j}} \mathbf{R}^{2}, j=1, \ldots, l$ so that $\left(\gamma\left(z_{1}\right), 1\right), \ldots,\left(\gamma\left(z_{k}\right), 1\right)$, $\left(\gamma\left(w_{1}\right), 1\right), \ldots,\left(\gamma\left(w_{l}\right), 1\right),\left(d \gamma\left(\xi_{1}\right), 0\right), \ldots,\left(d \gamma\left(\xi_{l}\right), 0\right)$ are linearly dependent. Thus, for some real numbers $a_{1}, \ldots, a_{k}, b_{1}, \ldots, b_{l}$, not all equal to zero, we have:

$$
\sum_{i=1}^{k} a_{i} \gamma\left(z_{i}\right)+\sum_{j=1}^{l} b_{j} \gamma\left(w_{j}\right)+\sum_{j=1}^{l} d \gamma_{w_{j}}\left(\xi_{j}\right)=0, \sum_{i=1}^{k} a_{i}+\sum_{j=1}^{l} b_{j}=0
$$

But $d \gamma_{w}(\xi)=\xi \gamma^{\prime}(w)$, where on the right side of the equation we view $\xi$ and $w$ as complex numbers and $\gamma$ as a map from $\mathbf{C}$ to $\mathbf{C}^{k+2 l-1}$, and this contradicts $(k, l)$-regularity in the complex sense, which we have already established.

Proof of Theorem 3.3 We will prove that $N_{k, l}\left(S^{1}\right)=k+2 l$ for even $\mathrm{k}$. The other two claims follow immediately from (2) and Proposition 3.2.

Lemma 4.2 Let $\gamma \subset R^{n}$ be a smooth curve with non-vanishing curvature vector $v$ at point $x$, and $H$ be a hyperplane, tangent to $\gamma$ at $x$ and transverse to $v$. Then, near $x$, the curve $\gamma$ doesn't cross $H$. 
Proof. Let $\gamma(t)$ be parametrized by arc length with $\gamma(0)=x$. Let $u=\gamma^{\prime}(0)$. Then

$$
\gamma(t)=x+t u+\frac{t^{2}}{2} v+\ldots
$$

We can assume $H$ to be the zero level hyperplane of a linear function $l$. Then, the nonvanishing curvature implies $l(v) \neq 0$, say $l(v)>0$. One has:

$$
l(\gamma(t))=l(x)+t l(u)+\frac{t^{2}}{2} l(v)+O\left(t^{3}\right)=\frac{t^{2}}{2} l(v)+O\left(t^{3}\right)>0
$$

for $t$ small enough, which proves the lemma.

Now we prove the lower bound by contradiction. Assume that $S^{1} \subset$ $\mathbf{R}^{k+2 l-1}$ as a $(k, l)$-regular submanifold. In order to arrive at a contradiction, we want to find a hyperplane in $\mathbf{R}^{k+2 l-1}$ that intersects $\gamma$ at $k$ points and touches at $l$ points, all of them distinct. Choose $l$ points $y_{j}$ where the curvature doesn't vanish. They must exist unless the curve $\gamma$ is straight. Let $W$ be the affine span of the tangent spaces (to $\gamma$ ) at these points. Let $v_{j}$ be the curvature vectors at $y_{j}$, and let $V_{j}$ be the affine span of $v_{j}$ and $W$. Note that $\operatorname{dim} V_{j} \leq 2 l$.

Assume that $k \geq 2$ (case $k=0$ holds because of Theorem 3.2). Note that $\gamma$ doesn't lie in $\cup_{j=1}^{l} V_{j}$, because then there would exist an open piece of this curve contained in one of the $V_{j}^{\prime}$ 's, which is impossible since $N_{k, l}(\mathbf{R}) \geq$ $k+2 l-1$. Choose generic $k-1$ points $x_{i} \in \gamma$ that belong to neither of $V_{j}$ and let $U$ be the affine span of $W$ and these points. Then $U$ is a hyperplane that is transverse to $\gamma$ at points $x_{i}$ (by their general position) and, by Lemma 4.2, $\gamma$ doesn't cross $U$ at points $y_{i}$. Thus we have an odd number of crossings of $\gamma$ and $U$, and since $\gamma$ is closed, there must be another one, say $x_{k} \in U \cap \gamma$. Therefore $\gamma$ is not $(k, l)$-regular.

\section{References}

[1] V. Arnold. On the number of flattening points on space curves. Amer. Math. Soc. Transl. (2) 171 (1996), 11-22.

[2] A. Barvinok. A course in convexity. AMS, Providence, 2002. 
[3] K. Borsuk. On the $k$-independent subsets of the Euclidean space and of the Hilbert Space. Bull. Acad. Pol. Sci. Cl.III 5, (1957),351-356.

[4] V. G. Boltyansky, S. S. Ryzhkov and Yu. A. Shashkin. On k-regular embeddings and their applications to the theory of approximation of functions. Uspekhi Mat. Nauk 15 (1960), no. 6 (96), 125-132; Amer. Math. Soc. Transl. (2) 28 (1963), 211-219.

[5] E. W. Cheney. Introduction to Approximation Theory, $2^{\text {nd }}$ Ed., Chelsea Publishing Company, New York, 1996.

[6] F. R. Cohen and D. Handel. $k$-regular embeddings of the plane, Proc. Amer. Math. Soc. 72 (1978), 201-204.

[7] M. Ghomi. Tangent bundle embeddings of manifolds in Euclidean space. Preprint.

[8] M. Ghomi. Nonexistence of skew loops on ellipsoids. Proc. Amer. Math. Soc., in print.

[9] M. Ghomi, B. Solomon. Skew loops and quadratic surfaces. Comment. Math. Helv. 77 (2002), 767-782.

[10] M. Ghomi, S. Tabachnikov. Totally skew embeddings of manifolds. Preprint, ArXiv: math.DG/0302288.

[11] D. Handel. Obstructions to 3-regular embeddings. Houston J. Math 5 (1979), 339-343.

[12] D. Handel. Approximation theory in the space of sections of a vector bundle. Trans. Amer. Math. Soc. 256 (1979), 383-394.

[13] D. Handel. Some existence and non-existence theorems for $k$-regular maps. Fund. Math. 109 (1980), 229-233.

[14] D. Handel. 2k-regular maps on smooth manifolds. Proc. Amer. Math. Soc. 124 (1996), 1609-1613.

[15] D. Handel, J. Segal. On $k$-regular embeddings of spaces in Euclidean space. Fund. Math. 106 (1980), 231-237. 
[16] J.-P. Sha, B. Solomon. No skew branes on non-degenerate hyperquadrics. Preprint, ArXiv: math.DG/0412197.

[17] G. Stojanovic, S. Tabachnikov. Non-existence of $n$-dimensional $T$ embedded discs in $\mathbf{R}^{2 n}$. Preprint, ArXiv: math.DG/0501323

[18] S. Tabachnikov. On skew loops, skew branes and quadratic hypersurfaces. Moscow Math. J. 3 (2003), 681-690.

[19] S. Tabachnikov, Y. Tyurina. Existence and non-existence of skew branes. Preprint, ArXiv: math.DG/0504484.

[20] V. A. Vassiliev. Complements of Discriminants of Smooth Maps: Topology and Applications, Revised Edition, AMS, Providence, 1992.

E-mail address: gordana@math.brown.edu; stojanov@math.psu.edu 